\title{
Moving Toward Improved Outcomes in Salvage Laryngectomy
}

\author{
Brett A. Miles, DDS, MD \\ Department of Otolaryngology Head and Neck Surgery, Icahn School of Medicine at Mount Sinai, New York, NY
}

Over the last 50 years there have been numerous advancements that have improved the surgical care of patients afflicted with head and neck cancer. Technological and technical advancements in surgery, such as minimally invasive endoscopic and robotic technology, as well as improvements in functional and anatomic imaging and in the delivery of radiotherapy and targeted chemotherapy, have allowed physicians to deliver dramatically improved oncologic care to a challenging patient population. Unfortunately, many of these advancements have had little impact on the outcomes related to salvage laryngectomy.

In the early 1990s, the results from several landmark trials validated the concept of larynx preservation in the treatment of advanced laryngeal squamous cell carcinoma. ${ }^{1-4}$ Nonsurgical therapy with concurrent chemoradiotherapy became the standard of care for all but the most advanced laryngeal squamous cell carcinomas. Currently, while the goal of organ preservation is being achieved in many patients, those who suffer a recurrence or residual disease after therapy generally undergo salvage laryngectomy as the 'gold standard' procedure, with acceptable oncologic results. Interestingly, the surgical technique for salvage laryngectomy has remained unchanged despite advancements in other aspects of head and neck surgery. Achieving a watertight suture closure remains an important aspect of the procedure, but it is widely recognized that the majority of complications (including salivary fistula) leading to prolonged hospital admissions and necessitating additional procedures are often not related to surgical technique in the majority of cases. More often, adverse outcomes are related to poor

\footnotetext{
(C) Society of Surgical Oncology 2017
}

First Received: 11 November 2017;

Published Online: 6 December 2017

B. A. Miles, DDS, MD

e-mail: brett.miles@mountsinai.org wound healing, infection, previous radiation, and complications related to medical comorbidities.

Perhaps the most impactful complication after salvage laryngectomy is salivary fistula, which often requires prolonged wound care during admission and increased morbidity for patients. In many cases, these fistula will require additional reconstructive surgery for correction and put the patient at risk for life-threatening carotid exposure with hemorrhage, and are certainly a burden on the healthcare system in terms of cost and resources. Current literature indicates the rates of postoperative fistula formation after salvage laryngectomy are approximately $30 \%$, with overall complication rates for the procedure reported at $67.5 \%$, shockingly high numbers for a procedure that has seen little technical advancements since first performed in 1873 by Theodor Billroth in Vienna but currently remains in widespread use. ${ }^{5}$

Unfortunately, today's surgeons continue to be plagued with salivary fistula after salvage laryngectomy, and several concepts have been evaluated to improve outcomes. Technical aspects related to the surgical procedure, such as salivary bypass tubes and free and pedicled muscle flaps, have been explored in order to reduce fistula rates; however, results are often difficult to interpret due to the heterogeneity of the patient population and surgical techniques utilized. ${ }^{6-9}$ Nevertheless, from a technical standpoint, vascularized reconstruction appears to play a role in fistula management. ${ }^{10}$ What has also become clear with these efforts is that technical aspects of surgery are not the only factors at play, and therefore technical alterations in the procedure are unlikely to resolve the issue of salivary fistula.

Therefore, it is helpful to conceptualize complications in salvage laryngectomy in order to identify modifiable risk factors that can be targeted for interventions to improve outcomes. Factors such as long-standing tobacco abuse and previous radiotherapy do not offer significant promise to alter outcomes as they are not modifiable at the time of 
surgery. Conversely, factors such as nutritional status, frailty, metabolic/endocrine abnormalities, or the use of alternative reconstructive techniques offer promise to improve outcomes in this patient population as these factors are modifiable in the perioperative period.

In the current issue of Annals of Surgical Oncology, Rosko et al. have provided an excellent analysis of 182 patients who underwent salvage laryngectomy, and noted a $47 \%$ fistula rate in hypothyroid patients compared with a $23 \%$ fistula rate in euthyroid patients, with hypothyroid patients exhibiting an 11.4-fold greater risk of developing a fistula that required reoperation when compared with euthyroid patients. Interestingly, $96 \%$ of patients were euthyroid prior to salvage surgery, indicating a causal relationship between salvage surgery and postoperative hypothyroidism, rather than other factors such as previous neck irradiation. Of note, the authors also reported an approximately $12.5 \%$ incremental increase in the absolute risk of fistula, with each doubling of the thyroid-stimulating hormone indicating the impact of the severity of hypothyroidism on outcomes.

It should be noted that the current work is not the first report linking hypothyroidism to salivary fistula in this setting. ${ }^{11,12}$ Nevertheless, this report offers some compelling data that has impact both on the perioperative management of the patient from an endocrine standpoint and perhaps also from a surgical technique perspective. First and foremost, routine removal of the thyroid gland should be avoided during laryngectomy procedures, and rarely is the thyroid gland itself involved with malignant disease, especially when bilateral lobes are considered. ${ }^{13,14}$ In addition, reconstructive surgeons should make efforts to spare the blood supply to the thyroid gland when selecting vessels for microvascular anastomosis when other options are available. It is unclear if the authors' finding that patients reconstructed with a vascularized flap had a 2.8fold higher incidence of developing postoperative hypothyroidism relates to this issue or represents a marker of more advanced disease requiring gland removal.

Perhaps the most important finding of this paper is quantifying the risk $(11.4 \times)$ of salivary fistula when surgical resection results in hypothyroidism. Certainly, hypothyroidism represents a modifiable risk factor both in terms of thoughtful surgical technique and gland preservation, as well as aggressive screening and medical management in the perioperative period. This data clearly demonstrates the fact that head and neck surgeons should make every effort to mitigate and control hypothyroidism in order to improve outcomes in this population.

\section{REFERENCES}

1. Department of Veterans Affairs Laryngeal Cancer Study Group, Wolf GT, Fisher SG, et al. Induction chemotherapy plus radiation compared with surgery plus radiation in patients with advanced laryngeal cancer. New Engl J med. 1991;324(24):1685-90.

2. Clayman GL, Weber RS, Guillamondegui O, et al. Laryngeal preservation for advanced laryngeal and hypopharyngeal cancers. Arch otolaryngol head neck surg. 1995;121(2):219-23.

3. Shirinian MH, Weber RS, Lippman SM, et al. Laryngeal preservation by induction chemotherapy plus radiotherapy in locally advanced head and neck cancer: the M. D. Anderson Cancer Center experience. Head neck. 1994;16(1):39-44.

4. Weber RS, Berkey BA, Forastiere A, et al. Outcome of salvage total laryngectomy following organ preservation therapy: the Radiation Therapy Oncology Group trial 91-11. Arch otolaryngol head neck surg. 2003;129(1):44-49.

5. Hasan Z, Dwivedi RC, Gunaratne DA, Virk SA, Palme CE, Riffat F. Systematic review and meta-analysis of the complications of salvage total laryngectomy. Eur J surg oncol. 2017;43(1):42-51.

6. Piazza C, Bon FD, Paderno A, et al. Fasciocutaneous free flaps for reconstruction of hypopharyngeal defects. Laryngoscope. 2017;127(12):2731-37.

7. Hone RWA, Rahman E, Wong G, et al. Do salivary bypass tubes lower the incidence of pharyngocutaneous fistula following total laryngectomy? a retrospective analysis of predictive factors using multivariate analysis. Eur arch oto-rhino-laryngol. 2017;274(4):1983-91.

8. Sharma S, Chaukar DA, Laskar SG, et al. Role of the pectoralis major myofascial flap in preventing pharyngocutaneous fistula following salvage laryngectomy. $J$ laryngol otol. 2016;130(9):860-64.

9. Patel UA, Moore BA, Wax M, et al. Impact of pharyngeal closure technique on fistula after salvage laryngectomy. JAMA otolaryngol head neck surg. 2013;139(11):1156-62.

10. Fung K, Teknos TN, Vandenberg CD, et al. Prevention of wound complications following salvage laryngectomy using free vascularized tissue. Head neck. 2007;29(5):425-30.

11. Bohannon IA, Carroll WR, Magnuson JS, Rosenthal EL. Closure of post-laryngectomy pharyngocutaneous fistulae. Head neck oncol. 2011;3:29.

12. White HN, Golden B, Sweeny L, Carroll WR, Magnuson JS, Rosenthal EL. Assessment and incidence of salivary leak following laryngectomy. Laryngoscope. 2012;122(8):1796-99.

13. Li SX, Polacco MA, Gosselin BJ, Harrington LX, Titus AJ, Paydarfar JA. Management of the thyroid gland during laryngectomy. J laryngol otol. 2017;131(8):740-44.

14. Mendelson AA, Al-Khatib TA, Julien M, Payne RJ, Black MJ, Hier MP. Thyroid gland management in total laryngectomy: meta-analysis and surgical recommendations. Otolaryngol head neck surg. 2009;140(3):298-05. 